\title{
Vulnerabilidade ao estresse, coping e burnout em educadoras de infância portuguesas ${ }^{1}$
}

\section{Vulnerability to stress, coping and burnout of Portuguese childhood educators}

\author{
Maria Regina Teixeira Ferreira Capelo ${ }^{2}$
}

\begin{abstract}
RESUMO
Esta investigação pretende analisar a relação entre vulnerabilidade ao estresse, estratégias de coping e burnout em educadoras de infância portuguesas. É um design quantitativo e transversal com uma amostra de 119 educadoras de infância portuguesas. Os instrumentos de recolha de dados são o Questionário de Vulnerabilidade ao Estresse, o Coping Job Scale e o Maslach Burnout Inventory. Os resultados indicam que, em situações de trabalho, o grupo percepciona-se não vulnerável ao estresse, utiliza maioritariamente estratégias de coping proactivas para lidar com situações indutoras de estresse e apresenta baixa prevalência de burnout; evidencia associações estatisticamente significativas entre vulnerabilidade ao estresse e as subescalas do burnout (exaustão emocional, despersonalização e realização pessoal); não revela relações estatisticamente significativas entre as estratégias de coping e burnout. Destes resultados, se infere a necessidade de averiguar as variáveis psicológicas, contextuais ou organizacionais, que estão a contribuir para a reduzida vulnerabilidade ao estresse e baixa prevalência de burnout neste grupo profissional.
\end{abstract}

Palavras-chave: vulnerabilidade ao estresse; coping; burnout; educadoras de infância.

DOI: $10.1590 / 0104-4060.49793$

1 A autora agradece a colaboração da Mestre Cristina Santos, pela distribuição do questionário, via e-mail.

2 Universidade de Lisboa. Faculdade de Letras. Centro de Literatura e Culturas Lusófonas e Europeias. Polo da Madeira. Funchal, Portugal. Campus Universitário da Penteada, 9020-105. E-mail: m.regina.capelo@gmail.com 


\begin{abstract}
This research intends to analyze the relationship between vulnerability to stress, coping and burnout strategies regarding Portuguese childhood educators. It has a quantitative and cross-sectional design with a sample of 119 Portuguese childhood educators. The data collection instruments are the Stress Vulnerability Questionnaire, the Coping Job Scale and the Maslach Burnout Inventory. The results indicate that, in work situations, the group perceived that it was not vulnerable to stress, it used mostly coping strategies that were proactive in dealing with stress-inducing situations and it had a low prevalence of burnout; it shows statistically significant associations between stress vulnerability and burnout subscales (emotional exhaustion, depersonalization and personal fulfillment); it does not reveal statistically significant relationships between coping strategies and burnout. From these results we inferred the need to ascertain the psychological, contextual or organizational variables which have been contributing to the reduced vulnerability to stress and low prevalence of burnout in this professional group.
\end{abstract}

Keywords: vulnerability to stress; coping; burnout; childhood educators.

\title{
Introdução
}

O estresse associado ao trabalho tem vindo a alcançar proporções epidémicas, convertendo-se num fenómeno importante e socialmente reconhecido (JESUS, 2001; MARTÍN; SALANOVA; PEIRÓ, 2003), pelas repercussões negativas para a saúde dos trabalhadores e, do ponto de vista económico, para as organizações (CORREIA; GOMES; MOREIRA, 2010; CHAMBEL, 2005; LÉGERON, 2008; MARTÍN; SALANOVA; PEIRÓ, 2003). Este fenómeno, presente na vida de todos os dias (VAZ SERRA, 2011), afeta a classe docente (ANTONIOU; POLYCHRONI; KOTRONI, 2009; CHIEN; KYRIACOU, 2004; KYRIACOU; SUTCLIFF, 1978; MARTINS, 2007; PINTO; SILVA; LIMA, 2003) e parece atingir com particular incidência os professores portugueses. (GOMES et al., 2012; GOMES et al., 2006; JESUS, 2005). Para tal, concorrem as mudanças societais e educacionais com implicações diretas ou indiretas no trabalho docente. (JESUS, 2005).

As potenciais fontes de estresse surtem efeitos diferenciados nos professores podendo constituir problemas para alguns, enquanto outros vivem satisfatoriamente a sua profissão. (JESUS, 2005). Neste sentido, Vaz Serra (2005, 2011) assevera que os seres humanos não são uniformemente vulneráveis ao 
estresse uma vez que a vulnerabilidade está relacionada com fatores biológicos, psicológicos, de personalidade e sociais.

De acordo com Vaz Serra $(2000,2005,2011)$, a pessoa vulnerável ao estresse tem um perfil caracterizado por pouca capacidade autoafirmativa, fraca tolerância à frustração, dificuldade em confrontar e resolver problemas, preocupação excessiva pelos acontecimentos quotidianos e marcada emocionalidade. Assim, qualquer episódio stressante obriga a nova adaptação por parte do indivíduo. (VAZ SERRA, 2005, 2011). Numa perspetiva transacional, Lazarus e Folkman (1984) referem que os esforços cognitivos e comportamentais realizados pelo indivíduo para lidar com os desafios internos ou externos, avaliados como ultrapassando os seus recursos, são representados pelo constructo coping.

O processo psicológico de coping pode ser desencadeado por fatores externos, tais como os estímulos físicos, as relações interpessoais e os acontecimentos de vida e/ou fatores internos, como os do conhecimento, os da emoção e os da personalidade. (LAZARUS; FOLKMAN, 1984). Por sua vez, o coping, entendido como um fenómeno adaptativo que contribui para a sobrevivência do indivíduo e para um adequado desempenho das suas atividades em diversas vertentes da vida (POCINHO; CAPELO, 2009), pode ser focado no problema ou nas emoções (LAZARUS; FOLKMAN, 1984; VAZ SERRA, 2005, 2011), e ainda na interação social. (VAZ SERRA, 2005, 2011).

Numa abordagem sobre as diferentes modalidades de coping, Vaz Serra (2011) realça que as estratégias focadas no problema tendem a ser utilizadas quando o estresse é percepcionado como pouco intenso e a situação resolúvel; que as estratégias focadas na emoção surgem quando o indivíduo conceptualiza que nada pode fazer para alterar a ameaça ou mudança ambiental; e, que as estratégias focadas na interação social referem-se à forma como a pessoa lida e mantém o relacionamento social em situações de estresse. Porém, o estímulo potencialmente estressante pode conduzir a diferentes respostas emocionais em diferentes indivíduos, dependendo da avaliação cognitiva, da situação e dos recursos (MARTÍN; SALANOVA; PEIRÓ, 2003), designadamente, a energia e saúde do indivíduo, os pensamentos de tipo positivo, a capacidade do indivíduo socializar (LAZARUS; FOLKMAN, 1984), as experiências passadas, as crenças, os valores, as características psicológicas como a personalidade, o locus de controlo, o autoconceito, a autoestima, o sentido de humor, o otimismo, etc. (RIBEIRO, 2009; VAZ SERRA, 2011) concorrem como variáveis diferenciadoras que influenciam os mecanismos de coping utilizados pelo indivíduo para lidarem com as fontes de estresse.

Latack (1986) conceptualiza as categorias de coping, tridimensionalmente, em controlo ou confronto, evitamento ou fuga e gestão de sintomas. As estratégias de controlo ou confronto consistem em ações e reavaliações cognitivas 
que são proactivas; as estratégias de evitamento ou fuga baseiam-se em ações e cognições que sugerem evitamento; e a gestão de sintomas, alicerçada em estratégias para gerir os sintomas. (LATACK, 1986; JESUS; PEREIRA, 1994). Embora Latack (1986) considere que, em ambiente profissional, as estratégias de controlo ou confronto produzem provavelmente melhores resultados, tendo concluído que estas estão associadas a baixo nível de ansiedade, menor tendência para abandono da profissão, maior grau de satisfação profissional e de necessidade de suporte social, diversos estudos sugerem inexistência de estratégias de coping modelares em relação às quais se possa referir que são eficazes ou ineficazes. (CAPELO, 2014; CAPELO; CALAÇA, 2016; JESUS; PEREIRA, 1994; POCINHO; CAPELO, 2009; QUIRINO, 2007; RIBEIRO, 2009; VAZ SERRA, 2011). Na verdade, a forma como o sujeito lida com o estresse não depende exclusivamente dos mecanismos de coping utilizados, mas dos processos psicológicos que, naquele momento, medeiam os efeitos das fontes de estresse sobre o bem-estar.

Quando as situações stressantes são vividas de forma prolongada, e o indivíduo sente que os seus recursos pessoais estão esgotados, desencadeiam de forma continuada respostas negativas que afetam a sua saúde física e mental. (CHAMBEL, 2005). Maslach, Schaufeli e Leiter (2001) revelam que muitos trabalhadores têm sido afetados por elevados níveis de desgaste físico e emocional. Esta problemática tem merecido grande atenção por parte dos investigadores organizacionais e estudada sob a designação de burnout.

A síndrome de burnout (metáfora da terra queimada), descrita clinicamente por Freudenber (1974), é definida como uma resposta ao estresse crónico no trabalho, expressa através de atitudes e sentimentos negativos em relação às pessoas, designadamente atitudes de despersonalização, experiência de exaustão emocional e falta de realização profissional. (CARLOTTO, 2011, MASLACH; JAKSON, 1981; MASLACH; JAKSON; LEITER, 1986; MASLACH; SHAUFELI, 1993). Esta síndrome multidimensional, que ocorre maioritariamente em profissionais de ajuda (MASLACH; SCHAUFELI; LEITER, 2001), segundo Maslach (2009), resulta da sobrecarga de trabalho, falta de controlo, sentimento de recompensa insuficiente, ausência de sentimento de comunidade, perceção de injustiça e conflito de valores. As pessoas com predisposição para sofrerem de burnout, na aceção de Vaz Serra (2011), são aquelas que ingressam numa atividade profissional cheias de sonhos e de boas intenções, esperançosas e dedicadas, mas que ao longo do tempo comprovam que não são apreciadas, deixando de ter significado aquilo que executam.

Embora exista extensa literatura acerca do estresse, do coping e do burnout em docentes, a investigação relacionada com educadoras de infância é escassa. O estudo de Gomes e Pereira (2008) sublinha que, na atualidade, ser 
“educador" apresenta constantes e complexos desafios que não se circunscrevem à criança, mas que englobam a assunção de papéis sociais múltiplos, como conceptualizador curricular, interventor/contextualizador, observador, avaliador, pesquisador, promotor da inteligibilidade da infância. Os resultados do referido estudo indicam que perante situações de estresse, os profissionais em início de carreira e em estágio utilizam principalmente estratégias de coping focadas na emoção do que as estratégias focadas no problema.

Atendendo à necessidade de melhor compreender os docentes que trabalham com crianças nos estádios mais precoces do ciclo vital, pretende-se com este estudo analisar a relação entre vulnerabilidade ao estresse, coping e burnout em educadoras de infância portuguesas.

\section{Método}

\section{Participantes}

Participaram neste estudo 119 educadoras de infância, que exercem a sua atividade com crianças (dos 0 aos 5 anos), nas creches, jardins de infância e estabelecimentos de educação pré-escolar, oficial, da ilha da Madeira, Portugal.

\section{Instrumentos}

Questionário de Vulnerabilidade ao Stress (23 QVS) (VAZ SERRA, 2000, 2005, 2011), de autoavaliação, com um total de 23 itens, cuja resposta varia entre 0 e 4, indica que quanto maior for a cotação, maior a probabilidade do indivíduo mostrar vulnerabilidade ao estresse, passando o valor 43 a constituir-se um ponto de corte acima do qual uma pessoa se revela vulnerável ao estresse. (VAZ SERRA, 2011). Quanto mais elevado é o valor do 23 QVS, menor tendência existe para o indivíduo adotar ações proactivas e maior propensão para sentir que não tem controlo sobre a situação. (QUIRINO, 2007). Além de uma análise unidimensional, contempla subescalas que, aparentemente, exprimem: perfeccionismo e intolerância à frustração; inibição e dependência funcional; carência de apoio social; condições de vida adversas; dramatização da existência; subjugação; e deprivação de afeto e rejeição. Na presente amostra, o instrumento apresenta valores de consistência interna aceitável $(\alpha=0,84$ na escala unidimensional; entre $\alpha=0,61$ e $\alpha=0,78$ nas subescalas).

Para avaliar as estratégias que os trabalhadores utilizam para lidarem com o estresse laboral utilizou-se a versão portuguesa do Coping Job Scale (CJS) 
de Latack (1986), traduzida e adaptada por Jesus e Pereira (1994), constituída por 52 itens, subdividida em estratégias de controlo ou confronto, estratégias de evitamento ou fuga e estratégias de gestão de sintomas. Esta escala é respondida tendo em conta 5 pontos de 1 (quase nunca faço isso) a 5 (quase sempre faço isso). Esta escala não avalia a eficácia das estratégias de coping na resolução de problemas, mas o grau em que o sujeito utiliza determinadas estratégias. (JESUS; PEREIRA, 1994; QUIRINO, 2007). O CJS apresenta caraterísticas psicométricas satisfatórias uma vez que o alfa de Cronbach varia entre $\alpha=0,68$ (fraco mas aceitável) na subescala estratégias de evitamento ou fuga e $\alpha=0,88$ (bom) na subescala estratégias de confronto ou controlo.

A avaliação inclui o Maslach Burnout Inventory (MBI) (MASLACH; JACKSON, 1986), traduzida e adaptada por Cruz e Melo (1996). Através desta escala de sete pontos ( 0 - nunca; 6 - todos os dias), constituída por 22 afirmações relacionadas com o trabalho, as participantes expressam a frequência com que cada sentimento ocorre, permitindo avaliar a síndrome de burnout. Segundo Gomes et al. (2006), Gomes et al. (2008) e Quirino (2007), níveis elevados de exaustão emocional e despersonalização e baixos scores de realização pessoal estão associados a altos índices de burnout. A adaptação portuguesa do MBI (MELO; GOMES; CRUZ, 1997) revela que scores elevados de burnout surgem quando os valores da exaustão emocional se encontram acima de 2,60, a despersonalização acima de 1,80 e a realização pessoal atinge níveis inferiores a 3,50. Os valores da consistência interna obtidos para a escala de MBI variam entre um mínimo de $\alpha=0,64$ (aceitável) na subescala despersonalização e um máximo de $\alpha=0,89$ (bom) na subescala exaustão emocional e oferecem adequação deste instrumento na avaliação do burnout em educadoras de infância.

\section{Procedimento}

Após a autorização da Secretaria Regional de Educação, foi enviado, via eletrónica, o protocolo de avaliação aos estabelecimentos de educação pré-escolar, creches e jardins de infância da ilha da Madeira para serem preenchidos. Os questionários estiveram disponíveis durante 30 dias, podendo ser preenchidos nos tempos livres dos participantes. No total a adesão foi de $21 \%$ do universo de educadores de infância em exercício na ilha da Madeira, Portugal. Foram salvaguardados os procedimentos éticos na investigação científica, designadamente, o consentimento informado, o sigilo e a confidencialidade dos dados. (FELDMAN, 2001). A participação foi voluntária.

Utilizou-se design quantitativo, transversal e correlacional. Realizou-se análises estatísticas na aplicação informática Statistical Package for Social Sciences (SPSS), versão 20. Verificou-se a consistência interna da amostra (alpha 
de Cronbach), o valor médio e o desvio padrão para cada uma das variáveis em estudo, determinou-se o nível médio de vulnerabilidade ao estresse e a prevalência do burnout. Calculou-se, ainda, o coeficiente de correlação de Pearson para determinar a relação entre a vulnerabilidade ao estresse, o coping e o burnout.

\section{Resultados}

\section{Vulnerabilidade ao estresse}

Os domínios da escala de vulnerabilidade ao estresse por ordem decrescente são "Perfeccionismo e intolerância à frustração" $(M=12,13 ; \mathrm{DP}=3,79)$, "subjugação" ( $M=6,73 ; \mathrm{DP}=2,73)$, "dramatização da existência" (M=6,18; $\mathrm{DP}=2,41)$, "inibição e dependência funcional" $(\mathrm{M}=5,60 ; \mathrm{DP}=3,03)$, "condições de vida adversas" ( $\mathrm{M}=3,30 ; \mathrm{DP}=1,89)$, "deprivação de afeto" $(M=3,24 ; \mathrm{DP}=1,96)$ e, por último, a "carência de apoio social" $(M=1,94 ; \mathrm{DP}=0,63)$. A análise da escala unidimensional reflecte que as educadoras de infância apresentam baixo score $(M=35 ; \mathrm{DP}=10,37)$, pois os valores médios são inferiores ao ponto de corte 43 traçado por Vaz Serra $(2000,2011)$, logo, é possível admitir que estas profissionais não revelam vulnerabilidade ao estresse.

\section{Coping}

O coping mais utilizado é o "controlo ou confronto" $(M=3,55 ; \mathrm{DP}=0,55)$, seguido do "evitamento ou fuga" $(M=3,04 ; \mathrm{DP}=0,48)$ e, por último, "gestão de sintomas" $(M=2,25 ; \mathrm{DP}=0,37)$. O resultado sugere que as educadoras de infância, tal como outros grupos docentes (JESUS, 2005; POCINHO; CAPELO, 2009; QUIRINO, 2007) recorrem, maioritariamente, a estratégias de enfrentamento proactivas, focadas na resolução de problemas, para lidarem com as situações indutoras de estresse.

\section{Burnout}

A análise descritiva sugere uma percentagem considerável de profissionais com valores baixos nas dimensões "exaustão emocional" $(M=1,73 ; \mathrm{DP}=1,18)$ - inferior a 2,60 $(23,50 \%)$ e "despersonalização" $(M=0,45$; DP=0,55) - inferior a 1,80 $(1,70 \%)$ e com valores elevados de "realização pessoal" $(M=27,90$; $\mathrm{DP}=4,85)$ - superiores a 3,50 $(94,10 \%)$. Apesar dos docentes incluírem um grupo 
profissional com sintomas de esgotamento superior ao de outros trabalhadores (HANAKKEN; BAKKER; SCHAUFELI, 2006), as educadoras de infância participantes neste estudo não indiciam perfil de profissionais burnout.

\section{Relação entre vulnerabilidade ao estresse e burnout}

A análise entre a escala unidimensional de vulnerabilidade ao estresse e o burnout, segundo autoavaliação das educadoras de infância participantes, revela forte associação positiva com a exaustão emocional $(r=0,55 ; \mathrm{p}<0,01)$, e com a despersonalização $(\mathrm{r}=0,45 ; \mathrm{p}<0,01)$; e negativa com a realização pessoal $(\mathrm{r}=-0,49 ; \mathrm{p}<0,01)$.

Existem correlações significativas positivas entre perfeccionismo e intolerância à frustração com a exaustão emocional $(r=0,45 ; \mathrm{p}<0,01)$ e com a despersonalização $(r=0,31 ; p<0,01)$ e negativa com a realização pessoal $(r=-0,41 ; p<0.01)$; inibição e dependência funcional e com a realização pessoal $(r=-0,41 ; \mathrm{p}<0,01)$, e positivamente com a exaustão emocional $(\mathrm{r}=0,45 ; \mathrm{p}<0,01)$ e despersonalização $(\mathrm{r}=0,31 ; \mathrm{p}<0,01)$; carência de apoio social correlaciona-se negativamente com a realização pessoal $(\mathrm{r}=-0,29 ; \mathrm{p}<0,01)$; condições de vida adversas correlaciona-se positivamente com a exaustão emocional $(\mathrm{r}=0,21 ; \mathrm{p}<0,05)$; dramatização da existência correlaciona-se positivamente com a exaustão emocional $(\mathrm{r}=0,34$; $\mathrm{p}<0,01)$ e com a despersonalização $(\mathrm{r}=0,30 ; \mathrm{p}<0,05)$, e negativamente com a realização pessoal $(\mathrm{r}=-0,26 ; \mathrm{p}<0,01)$; subjugação correlaciona-se negativamente com a realização pessoal $(\mathrm{r}=-0,29 ; \mathrm{p}<0,01)$, e positivamente com a exaustão emocional ( $r=0,27 ; p<0,01)$, e a despersonalização $(r=0,27 ; p<0,01)$; deprivação de afeto continua a apresentar correlação positiva com a exaustão emocional $(r=0,49 ; p<0,01)$ e a despersonalização $(r=0,49 ; p<0,01)$ e negativa com a realização pessoal $(r=-0,44 ; \mathrm{p}<0,01)$.

Os resultados evidenciam associação entre a vulnerabilidade ao estresse tanto a nível da escala unidimensional, bem como das respetivas subescalas e o burnout. Logo, quanto maior for o nível de vulnerabilidade ao estresse, maior será o nível de exaustão emocional, maior o nível de despersonalização e menor nível de realização pessoal.

\section{Relação entre vulnerabilidade ao estresse e estratégias de coping}

Entre a vulnerabilidade ao estresse (unidimensional) e as estratégias de coping utilizadas pelas educadoras de infância destaca-se correlação negativa com as estratégias de gestão de sintomas $(r=-0,20 ; p<0,05)$, bem como correlações negativas entre a dimensão subjugação e as estratégias de gestão de sintomas $(\mathrm{r}=-0,19 ; \mathrm{p}<0,05)$, entre a dimensão inibição e dependência funcional com as 
estratégias de controlo ou confronto $(\mathrm{r}=-0,23 ; \mathrm{p}<0,05)$ e com as estratégias de gestão de sintomas $(\mathrm{r}=-19 ; \mathrm{p}<0,05)$. Isto significa que quanto mais vulnerável o indivíduo se perceciona, menor é a tendência para adoção de estratégias de confronto proactivas e maior tendência para sentir que não tem controlo sobre a situação.

\section{Relação entre estratégias de coping e burnout}

Nesta amostra, as dimensões do coping não apresentam correlações estatisticamente significativas com qualquer subescala do burnout, podendo inferir-se que processos psicológicos utilizados no momento, não contemplados neste estudo, poderão mediar os efeitos das fontes de estresse sobre a perceção de bem-estar e influenciar os níveis de exaustão emocional, de despersonalização e de realização pessoal das educadoras de infância.

\section{Discussão}

O estudo do estresse adquiriu relevância e reconhecimento social, nas últimas décadas, principalmente nas sociedades desenvolvidas, sobretudo pelos efeitos psicológicos, fisiológicos e comportamentais que produzem nos indivíduos e nas organizações, associando-os de maneira frequente ao trabalho. (CHAMBEL, 2005; JESUS, 2005; MARTÍN; SALANOVA; PEIRÓ, 2003; PEIRÓ, 2001; VAZ SERRA, 2005, 2011). Não obstante o estresse ser uma realidade socialmente construída, Peiró (2001) recomenda o seu estudo como um fenómeno coletivo. No caso particular dos profissionais da Educação de Infância, os estudos são escassos, razão pela qual surge esta pesquisa que pretende analisar a relação entre vulnerabilidade ao estresse, estratégias de coping e burnout em educadoras de infância portuguesas.

Foi possível verificar que o grupo participante, exclusivamente feminino, é constituído por 119 educadoras de infância. Esta evidência reproduz a ideia que a prática docente é uma atribuição feminina (VIANNA, 2013) e revela a necessidade de um estudo aprofundado quando se discute um projeto ético-profissional para este grupo profissional. (ROSA, 2011).

Neste estudo, os valores médios de vulnerabilidade ao estresse são baixos $(\mathrm{M}=35)$, inferiores ao valor 43 que constitui o "ponto de corte" proposto por Vaz Serra (2000). Os resultados do presente estudo revelam média baixa em todos os 
fatores da vulnerabilidade ao estresse, sendo o "perfeccionismo e intolerância à frustração" a variável com média mais elevada $(\mathrm{M}=12,13)$.

De acordo com Vaz Serra $(2000,2011)$, as educadoras de infância participantes não revelam vulnerabilidade ao estresse, daí a necessidade de se indagar as variáveis moderadoras que estão a influenciar a baixa vulnerabilidade ao estresse neste grupo profissional.

Também pertinente é o fato destas profissionais recorrerem, maioritariamente, a estratégias de coping proactivas $(\mathrm{M}=3,55)$ para lidarem com os desafios do quotidiano laboral, seguidas das estratégias de evitamento ou fuga $(M=3,04)$ e das estratégias de gestão de sintomas $(M=2,25)$. Resultados análogos foram obtidos por Capelo (2014), Capelo e Calaça (2016), Jesus e Pereira (1994), Pocinho e Capelo (2009) e Quirino (2007) com amostras de professores portugueses. Também o estudo exploratório de Capelo, Pocinho e Santos (2013) coloca em destaque as seguintes estratégias adaptativas referidas pelas educadoras de infância: estabelecer boas relações com os colegas e com os pais/encarregados de educação; gerir as emoções; comunicar com os pares, hierarquia e pais/encarregados de educação; evitar conflitos; garantir a segurança das crianças; programar e avaliar atempadamente as atividades pedagógicas são as estratégias adaptativas mais requeridas.

Latack (1986) preconizou que, em ambiente laboral, as estratégias de controlo ou confronto provavelmente produzem melhores resultados. No entanto, na aceção de Capelo (2014), Jesus e Pereira (1994), Pocinho e Capelo (2009), Quirino (2007), Ribeiro (2009) e Vaz Serra (2011), a eficácia das estratégias de coping irá depender dos processos psicológicos que medeiam os efeitos dos desafios stressantes sobre o bem-estar do indivíduo. Porém, quando o indivíduo avalia que as exigências requeridas pelo trabalho superam os seus recursos, e vive estas situações de modo prolongado, desencadeia respostas negativas, emocionais e/ou fisiológicas e/ou comportamentais que afetam a sua saúde física e mental (CHAMBEL, 2005; MASLACH; SCHAUFELI; LEITER, 2001) e assoma o burnout. Sendo o burnout um fenómeno psicossocial relacionado com o contexto laboral, caraterizado pela exaustão emocional, a despersonalização e a falta de realização pessoal, que acomete trabalhadores de forma direta e emocional e pode trazer consequências físicas, psíquicas, comportamentais e defensivas (FRANÇA et al. 2014), requere por parte da organização a assunção de medidas profiláticas e/ou reparadoras, e pelo indivíduo, o recurso a estratégias de enfrentamento proactivas, para combater a síndrome e minimizar os seus efeitos.

No que concerne à prevalência de burnout, ressalta os baixos scores de exaustão emocional $(M=1,75)$ e na despersonalização $(M=0,45)$ e elevados scores na escala de realização pessoal $(\mathrm{M}=27,89)$. Estes resultados são di- 
ferentes dos estipulados por Maslach, Jackson e Leiter (1986), que sugerem valores acima de 2,6 na dimensão exaustão emocional, valores acima de 1,8 na dimensão despersonalização e valores inferiores a 3,5 na dimensão realização profissional para caracterizar o perfil burnout. Importa realçar que nenhuma das profissionais participantes pontuou scores elevados, simultaneamente, nas dimensões "exaustão emocional" (23,5\%), "despersonalização" $(1,7 \%)$ e "baixa realização pessoal" (5,9\%), logo não é possível identificar um perfil burnout nestas educadoras de infância. Mas, uma vez que profissionais em situação de exaustão emocional, despersonalização e baixa realização pessoal, provavelmente, não contribuíram para este estudo, é necessária precaução nas ilações que se possam extrair dos resultados obtidos.

Quanto à relação entre a vulnerabilidade ao estresse e o nível de burnout, os coeficientes de correlação de Pearson evidenciam associações estatisticamente significativas entre vulnerabilidade ao estresse e as subescalas do burnout (exaustão emocional, despersonalização e realização pessoal). Estes resultados corroboram a hipótese que "existe associação positiva entre a vulnerabilidade ao estresse e o burnout", portanto, quanto maior a vulnerabilidade ao estresse, mais elevado é o burnout. (VAZ SERRA, 2011).

Os resultados mostram também uma associação negativa com as estratégias de gestão de sintomas, o que sugere que as educadoras que utilizam menos estratégias de gestão de sintomas são mais vulneráveis ao estresse. Verificou-se, ainda, diversas associações significativas positivas entre os fatores da vulnerabilidade ao estresse e as dimensões do burnout, sendo positivas com a exaustão emocional e a despersonalização e negativas com a realização profissional.

Neste estudo, não foram encontradas evidências de que existe associação entre as estratégias de coping e o burnout. A este respeito, não se descobriu suporte na literatura consultada que relacione as estratégias de coping e o burnout na população em apreço, criando espaço para novas interrogações. Porém, o estudo de Quirino (2007), realizado com professores do $3^{\circ}$ ciclo do ensino básico, revela que os professores que recorrem a estratégias de controlo na sua atividade profissional evidenciam menor exaustão emocional. Também Pinto, Silva e Lima (2005) verificaram que o coping centrado na resolução de problemas é um preditor significativo de baixos scores de exaustão emocional.

Os dados foram recolhidos nos estabelecimentos de educação de infância de meio urbano e rural, numa específica região insular, logo as inferências efetuadas não são passíveis de generalização. Mostram um grupo profissional, exclusivamente feminino, com reduzida vulnerabilidade ao estresse e baixa prevalência de burnout permitindo depreender que as educadoras de infância participantes neste estudo vivem positivamente a sua profissão. (JESUS, 2005). Neste sentido, Rosa (2011) verifica que a profissão docente nos anos iniciais leva consigo estereótipos 
criados acerca do género e incorporados na representação dos próprios docentes. Esta evidência denuncia a necessidade de compreender a reprodução da ideia de que a prática docente com crianças é uma atribuição feminina.

A participação foi livre e informada. Contudo, os dados não asseguram a inexistência de profissionais vulneráveis ao estresse e com perfil burnout no contexto em estudo. Mas, tendo em consideração que o estresse é dinâmico, localizado, em avaliação ou em resposta (emocionais, fisiológicas, comportamentais), contextualizado e interativo; que o coping se refere à transação entre as fontes de estresse e a pessoa afetada (LAZARUS; FOLKMAN, 1984); que o estresse afeta diversas pessoas, com diferentes personalidades e em diferentes etapas da vida (VAZ SERRA, 2011); que as diferenças individuais podem ter impacto no nível de estresse e no enfrentamento, importa compreender os seus efeitos, ao longo do tempo, na vida dos indivíduos. (SEGERSTROM; O'CONNOR, 2012).

Em suma, a investigação revela que existe associação positiva entre a vulnerabilidade ao estresse e o burnout, mas não prova a existência de associação negativa entre as estratégias de coping e burnout. Também conduz à necessidade de aprofundamento do tema numa amostra mais alargada que permita esclarecer em maior profundidade a relação entre a vulnerabilidade ao estresse, as estratégias de coping e o burnout, num grupo docente, feminizado, dedicado profissionalmente a pessoas nos escalões mais precoces dos estágios da vida humana - a Educação da Infância. Sugere-se, assim, a realização de novos estudos, com uma amostra mais alargada, que englobe o género masculino, variáveis sociodemográficas, profissionais e inclua outras variáveis moderadoras, como o suporte social, as crenças de autoeficácia, a motivação, o otimismo, o bem-estar psicológico, a qualidade de vida, etc., em diferentes contextos, que contribuam para a compreensão mais aprofundada deste grupo profissional.

\section{REFERÊNCIAS}

ANTONIOU, A.; POLYCHRONI, F.; KOTRONI, C. Working with students with special educational needs in Greece: Teachers stressors and coping strategies. International Journal of Special Education, v. 24, n. 1, p. 100-111, 2009.

CAPELO, R. Estrés, coping y autoeficácia. Estresores más frequentes y estratégias personales para mejorar el bienestar docente. Saarbrücken: Publicia, 2014.

CAPELO, R.; CALAÇA, Z. O poder preditivo do coping e do engagement no estresse laboral dos professores do ensino especial. Informes Psicológicos, v. 16, n. 2, p. 13-25, 2016. 
CAPELO, R.; POCINHO, M.; SANTOS, C. Estresse e Coping em educadoras de infância portuguesas: um estudo exploratório. Trabajo: Revista andaluza de relaciones laborales, U. Huelva, v. 28, p. 69-81, 2013.

CARLOTTO, M. Síndrome de burnout em professores: prevalência e factores associados. Psicologia: Teoria e Pesquisa, v. 27, n. 4, p. 403-410, out./dez. 2011.

CHAMBEL, M. Stess e bem-estar nas organizações. In: PINTO, A.; SILVA, A. (Coord.). Stress e bem-estar. Lisboa: Climepsi Editores, 2005. p. 105-134.

CHIEN, P.; KYRIACOU, C. Teacher stress in Taiwanese primary schools. Journal of Educational Enquiry, v. 5, n. 2, p. 89-104, 2004.

CORREIA, T.; GOMES, R.; MOREIRA, S. Estresse ocupacional em professores do ensino básico: Um estudo sobre as diferenças pessoais e profissionais. In: SIMPÓSIO NACIONAL DE INVESTIGAÇÃO EM PSICOLOGIA, VII., 2010, Braga. Actas... Braga: Universidade do Minho, 2010. p. 1477-1493.

CRUZ, J.; MELO, B. Stress e "burnout" nos psicólogos: desenvolvimento e características psicométricas de instrumentos de avaliação. Relatório de investigação não publicado. Braga: Universidade do Minho, 1996.

ESTEVE, J. Mudanças sociais e mudanças na educação: da educação de elite à educação de massas. In: NÓVOA, A. (Org.). Profissão Professor. Porto: Porto Editora, 1995. p. 95-124.

FELDMAN, R. Compreender a Psicologia. Amadora: McGraw-Hill, 2001.

FRANÇA, T.; OLIVEIRA, A.; LIMA, L.; MELO, J.; SILVA, R. Síndrome de burnout: Characterístics, diagnosis, risk factos and prevention. Revista de Enfermagem, v. 8, n. 10, p. 3539-3546, 2014.

FRANÇA, T.; OLIVEIRA, A.; MELO, J.; SILVA, R. Síndrome de burnout: características, fatores de risco e prevenção. Revista de Enfermagem UFPE Online, v. 8, n. 10, p. 3539-3546, 2014.

FREUDENBERGER, H. Staff burnout. Journal of Social Issues, v. 30, v, 1, p. 159-165, Winter, 1974.

GOMES, R.; PEREIRA, A. Estratégias de coping em educadores de infância portugueses. Revista Semestral da Associação Brasileira de Psicologia Escolar e Educacional (ABRAPEE), v. 12, n. 2, p. 319-326, jul./dez. 2008.

GOMES, R.; CABANELAS, S.; MACEDO, V.; PINTO, C.; PINHEIRO, L. Stress, saúde física, satisfação e "burnout" em profissionais de saúde. Análise das diferenças em função do sexo, estado civil e agregado familiar. In: PEREIRA, M. G.; SIMÕES, C.; MCINTYRE, T. (Eds.). Actas do II Congresso família, saúde e doenças: modelos, investigação e prática em diferentes contextos de saúde. Braga: Universidade do Minho, 2008. v. 4, p. 178-192. 
GOMES, R.; PEIXOTO, A.; PACHECO, R.; SILVA, M. Estresse ocupacional e alteração do estatuto da carreira docente. Educação e Pesquisa, v. 38, n. 2, p. 357-371, abr./ jun. 2012.

GOMES, R.; SILVA, M.; MOURISCO, S.; SILVA, S.; MOTA, A.; MONTENEGRO, N. Problemas e desafios no exercício da atividade docente: um estudo sobre o estresse, burnout, saúde física e satisfação profissional em professores do $3 .^{\circ}$ ciclo e ensino secundário. Revista Portuguesa de Educação, v. 19, n. 1, p. 67-93, 2006.

GUILLÉN GESTOSO, C. Estratégias de negociação. Mangualde: Edições Pedago, 2005.

HANAKKEN, J.; BAKKER, A.; SCHAUFELI, W. Burnout and Work engagement among teachers. Journal of School Psychology, v. 43, p. 495-513, 2006.

JESUS, S. Como prevenir e resolver o stress dos professores e a indisciplina dos alunos. Porto: ASA Editores II, S. A., 2001.

JESUS, S. N. Bem-estar docente. Perspectivas para superar o mal-estar dos professores. In: PINTO, A.; SILVA, A. (Coord.). Stress e bem-estar. Modelos e domínios de aplicação. Lisboa: Climepsi Editores, 2005. p. 167-184.

JESUS, S.; PEREIRA, A. Estudo das estratégias de "coping" utilizadas pelos professores. In: SEMINÁRIO A COMPONENTE DA PSICOLOGIA NA FORMAÇÃO DE PROFESSORES E OUTROS AGENTES EDUCATIVOS, 5., 1994, Évora. Actas... Universidade de Évora, 1994. p. 243-268.

KYRIACOU, C.; SUTCLIFF, J. Teacher Stress: Prevalence, Sources, and Symptons. Bristish Journal of Educational Psychology, v. 48, n. 2, p. 159-167, 1978.

LATACK, J. Coping with job stress: measures and future directions for scale development. Journal of Applied Psychology, v. 71, n. 3, p. 377-385, Aug. 1986.

LAZARUS, R.; FOLKMAN, S. Stress, appraisal and coping. New York: Springer, 1984.

LÉGERON, P. Le stress professionnel. L'Information Psychiatrique, v. 84, n. 9, p. 809-820, 2008.

MARTÍN, P.; SALANOVA, M.; PEIRÓ, J. El estrés laboral. Un concepto cajón-de-sastre? Proyecto Social: Revista de relaciones laborales, v. 10-11, p. 167-185, 2003.

MARTINS, M. Sintomas de stress em professores brasileiros. Revista Lusófona de Educação, 10, p. 109-128, 2007.

MASLACH, C. Entendendo o burnout (Understanding burnout). Ciência e Trabajo, v. 11, n. 32, p. 37-43, 2009.

MASLACH, C.; JAKSON, S. The measurement of experienced burnout. Journal of Occupational Behavior, v. 2, p. 99-113, 1981.

MASLACH, C.; JACKSON, S.; LEITER, M. Maslach Burnout Inventory. Consulting Psychologists Press, Palo Alto, C. A, 1986. 
MASLACH, C.; SCHAUFELI, W. Historical and conceptual development of burnout. In: SCHAUFELI, W. B.; MASLACH, C.; MAREK, T. (Eds.). Profesional Burnout: Recent Development in Theory and Research. Washingtom, DC: Taylor \& Francis, 1993. p. 1-16.

MASLACH, C.; SCHAUFELI, W.; LEITER, M. Defining the construct: What is burnout? Rev. Psychol, v. 52, p. 397-422, 2001.

MELO, B.; GOMES, A.; CRUZ, J. Stress ocupacional em profissionais de saúde e do ensino. Psicologia, Teoria, Investigação e Prática, v. 2, p. 53-71, 1997.

PEIRÓ, J. El estrés laboral: una perspectiva individual y colectiva. Prevención, trabajo y salud: Revista del Instituto Nacional de Seguridad e Higiene en el Trabajo, v. 13, p. 13-38, 2001.

PINTO, A.; SILVA, A.; LIMA, M. Como lidam os professores com o stress profissional? Coping e burnout em professores portugueses.Proform@ronline [revista on-line], v. 7, n. 1,2005 .

PINTO, A.; SILVA, A.; LIMA, M. Stress profissional em professores portugueses: incidência, preditores e reação de burnout. Psychologica, v. 33, p. 181-194, 2003.

POCINHO, M.; CAPELO, R. Vulnerabilidade ao estresse, coping e auto-eficácia em professores portugueses. Educação e Pesquisa, v. 35, n. 2, p. 351-367, 2009.

QUIRINO, A. Stress, coping e burnout em professores do $3{ }^{\circ}$ Ciclo. Dissertação (Mestrado em Psicologia da Saúde) - Universidade do Algarve, Faro, Universidade do Algarve, 2007.

RIBEIRO, J. Coping: estratégias de gestão de estresse. Health \& Wellness, p. 28-20, 2009.

ROSA, R. Feminização do Magistério: representações e espaço docente. Revista Pandora Brasil, edição especial 4 - Cultura e Materialidade Escolar, n. 1, p. 1-19, 2011.

SEGERSTROM, S.; O'CONNOR, D. Stress, health and illness: Four challenges for the future. Psychol Health, v. 27, n. 2, p. 128-140, 2012.

VAZ SERRA, A. As múltiplas facetas do stress. In: PINTO, A.; SILVA, A. (Coord.). Stress e bem-estar. Lisboa: Climepsi Editores, 2005. p. 17-42.

VAZ SERRA, A. Construção de uma escala para avaliar a vulnerabilidade ao stress: a 23 QVS. Psiquiatria Clínica, v. 21, n. 4, p. 261-278, 2000.

VAZ SERRA, A. O stress na vida de todos os dias. Coimbra: Adriano Vaz Serra Editor, 2011.

VIANNA, C. A feminização do magistério na educação básica e os desafios para a prática e a identidade coletiva docente. In: YNANNOULAS, S. C. (Org.). Trabalhadoras: análise da feminização das profissões e ocupações. Brasília. Abaré, 2013. p. 159-180.

Texto recebido em 16 de dezembro de 2016.

Texto aprovado em 17 de dezembro de 2016. 
